\title{
Postoperative Fracture Healing Effects of Locking Compression Plate for the Treatment of Limb Fractures
}

\author{
Bin Zhao' ${ }^{1}$, Jingli Dou ${ }^{2}$, Rongcai Zhang1, Mingming Wang ${ }^{1 *}$ \\ ${ }^{1}$ Department of Orthopedic Surgery, Binzhou People's Hospital, 515 Huanghe Qilu, Binzhou 256600, Shandong Province, \\ China
}

${ }^{2}$ Operating Room, Binzhou People’s Hospital, 515 Huanghe Qilu, Binzhou 256600, Shandong Province, China

\begin{abstract}
[Abstract] Objective: To study the effects of applying locking compression plates in the treatment of patients with limb fractures on postoperative fracture healing. Methods: 115 patients with limb fractures who were treated in our hospital from November 2019 to November 2020 were selected. In order to study the effective treatment method, the random-number table method was used in this study to divide the patients into two groups, namely the experimental group and the control group, and the locking compression plate treatment method and the pure plate and screw internal fixation treatment method were administered respectively to study their clinical application effects. Results: Compared with the control group, patients in the experimental group had a lower incidence of complications, shorter hospitalizations and shorter recovery time. Meanwhile, the experimental group had a better quality of recovery, and all data were significantly different from those of the control group, $\mathrm{P}<0.05$, the intervention effect of the experimental group was better. Conclusion: The application of the locking compression plate in the treatment of patients with limb fractures is more conducive to promoting the postoperative healing of the patients' fractures, reducing the incidence of postoperative complications, and promoting the rapid recovery of patients, which has positive significance for clinical development.
\end{abstract}

Key words: Locking compression plate treatment method; Simple plate and screw internal fixation treatment method; Patients with limb fractures

Publication date: May, 2021; Publication online: 31 May, 2021

*Corresponding author: Mingming Wang, zbin1356307@163.com

Limb fractures are usually caused by factors such as traffic accidents, mechanical injuries, and falls from height. After fracture occurs, the patients' injuries are usually more serious, movement is limited and there will be many complications. The occurrence of the fracture will cause certain damage to the adjacent organs, blood vessels and nerves around the fracture, and timely and effective treatment is required for the patients. If handled improperly, it will easily affect the quality of life of the patients severely ${ }^{[1]}$. From the perspective of clinical development status, most of the treatments for patients are the application of conventional plaster and splint external fixation methods, but the effects of these methods are not significant enough, therefore effective treatment strategies need to be explored clinically. On this basis, this study applied the locking compression plate treatment method to explore the difference between the effects of this treatment method and the simple plate and screw internal fixation method. The analysis report is as follows.

\section{Patient information and research methods}

\subsection{Information analysis}

This research work was selected to be carried out in our hospital from November 2019 to November 2020. All the patients suffered from limb fractures, and there were 115 cases. In order to study the intervention effects of different 
treatment methods, this study divided the patients into two groups, where the patients were treated with simple screw internal fixation method (control group) and the locking compression plate treatment method (experimental group) respectively, and the number of patients were 60 and 55 respectively. In the experimental group, there were 27 males and 28 females. The maximum and minimum ages of the patients were 70 and 18 years old respectively, and the average age was $(46.23 \pm 3.43)$ years. The number of males and females in the control group were 28 and 32 respectively. The upper limit and the lower limit of the patients' age were 71 and 19 years old respectively, and the average age was $(45.84 \pm 4.03)$ years. The general information of the two groups of patients is relatively similar and inter-group comparison can be carried out ${ }^{[2]}$.

Inclusion criteria: The patient was diagnosed with limb fractures after X-ray examination; no contraindication; after the patients had been informed of the purpose and significance of the research work, they all participated in the research voluntarily; the research work gained the approval from the hospital ethics committee.

Exclusion criteria: poor compliance of the patient; the patient has a history of mental illness; the patient developed complication of infection; the time from fracture to surgery was more than 18 hours.

\subsection{Research methods}

In this study, patients in the control group were treated with simple plate and screw internal fixation. In the specific operation process, the patients were first given epidural anesthesia, and an incision at the proximal end of the fracture with a length of about 2 to $3 \mathrm{~cm}$ was made, which then promoted the effective incision of their skin and the subcutaneous tissue to promote the effective exposure of the fractured end under the surgical view. The bone fragments and haemorrhage were removed, and then fracture reduction was carried out, the steel plate was placed on the surface of the broken bone, and transmission imaging with $\mathrm{C}$-arm $\mathrm{X}$-ray was performed to achieve effective adjustment of the position of the steel plate to ensure that it is in the best position. Meanwhile, it is necessary to insert the screws at the proximal and distal ends of the fracture. The number of insertions is $2 \sim 4$. The fracture fixation and reduction of the patient were observed under C-arm X-ray fluoroscopy. After confirmation, the incision was sutured layer by layer, and plaster external fixation should be applied after the surgery until the patient's fracture heals.

The experimental group was treated with a locking compression plate. In the actual operation, the patient needed to be given epidural anesthesia, and then an incision with a length of 2 to $3 \mathrm{~cm}$ was made at the proximal end to promote the separation of the tissue, shaping it like a tunnel mainly outside the periosteum at the subfascia, and the steel plate was inserted with the help of a C-arm X-ray machine under fluoroscopy, and the position of the steel plate was effectively adjusted. Then, one screw was inserted at the proximal and distal end of the plate each. After confirming the fracture reduction, the screws were placed again at the proximal and distal ends. The number of insertions is $2 \sim 4$. Then the incision was sutured and the external fixation was performed after plastering. Patients were instructed to undergo early functional rehabilitation exercises.

\subsection{Observation indicators}

The fracture recovery time and hospitalization time of the two groups were compared and analyzed, and the incidence of complications and fracture recovery status of the two groups were compared.

\subsection{Statistical method}

The software SPSS20.0 was used for the statistical analysis of the data, the analysis of measurement data ( $X_{ \pm s}$ ) was verified by t-test, while the calculation and comparison of the count data $(\mathrm{n}, \%)$ was verified by $\mathrm{X}^{2}$ test. When the result is shown to be $\mathrm{P}<0.05$, it means that the comparison of the study is statistically significant.

\section{Results}

\subsection{Recovery and hospitalization time}

The recovery and hospitalization time of the patients in the experimental group were shorter than those in the control group, and the differences between the groups were all shown to be $\mathrm{P}<0.05$, which are statistically significant (Table 1).

Table 1. Comparison of recovery and hospitalization time between the two groups $\left(\bar{X}_{ \pm \mathrm{s}}\right)$

\begin{tabular}{ccc}
\hline Group & Recovery Time (week) & Hospitalization Time (day) \\
\hline Experimental $(\mathrm{n}=55)$ & $13.23 \pm 1.09$ & $9.03 \pm 1.02$ \\
Control $(\mathrm{n}=60)$ & $20.43 \pm 2.23$ & $14.69 \pm 1.23$
\end{tabular}




\subsection{Incidence of complications}

The incidence of complications in the experimental group was $7.27 \%$, and the incidence of complications in the control group was $20.00 \%$. The difference was relatively large, and the incidence of complications in the experimental group was lower (Table 2).

Table 2. Comparison on the incidence of complications between the two groups [n(\%)]

\begin{tabular}{ccccc}
\hline Group & Displacement & Infection & Internal Fixation Loosening & Incidence \\
\hline Experimental $(\mathrm{n}=55)$ & $1(1.82)$ & $2(3.64)$ & $1(1.82)$ & $4(7.27)$ \\
Control $(\mathrm{n}=60)$ & $3(5.00)$ & $5(8.33)$ & $4(6.67)$ & $12(20.00)$ \\
$\mathrm{X}^{2}$ & & & 7.496 \\
$\mathrm{P}$ & & & $<0.05$ \\
\hline
\end{tabular}

\subsection{Quality of recovery}

The quality of recovery of the two groups of patients were $94.55 \%$ and $81.67 \%$, respectively, and the difference between the groups was relatively large. The quality of recovery of the experimental group was higher, which is statistically significant (Table 3).

Table 3. Comparison of the quality of recovery in patients between the two groups $[\mathrm{n}(\%)]$

\begin{tabular}{ccccc}
\hline Group & Excellent & Good & Bad & Quality \\
\hline Experimental $(\mathrm{n}=55)$ & $37(67.27)$ & $15(27.28)$ & $3(45.45)$ & $52(94.55)$ \\
Control $(\mathrm{n}=60)$ & $29(48.33)$ & $20(33.33)$ & $11(19.34)$ & $49(81.67)$ \\
$\mathrm{X}^{2}$ & & & & 13.956 \\
$\mathrm{P}$ & & & & $<0.05$ \\
\hline
\end{tabular}

\section{Discussion}

Limb fractures are rather common in clinical practice and belong to the category of fractures. The patients have the characteristics of serious injuries and more complicated conditions $^{[3]}$. In the past, clinical treatment usually took a long time, and the incidence of complications was relatively high, which caused serious adverse effects on patients ${ }^{[4]}$. Judging from the current development status, most surgical methods are applied in clinical practice when treating patients. With the continuous advancement of medical technology, more and more surgical methods have become available. The selection of the best surgical method for patients is the main factor affecting the promotion of recovery in patients. The traditional treatment method of steel plate internal fixation has the characteristics of simple operation and being economical ${ }^{[5]}$. However, postoperative wound healing in patients is generally relatively slow, and it is prone to complications such as nonunion of bones and incision infection, which will adversely affect the patient's functional recovery. When the locking compression plate treatment method is applied, it not only brings relatively minor trauma, but also does not adversely affect the patient's internal and external blood supply, and its safety in clinical application is relatively prominent ${ }^{[6]}$.

In this study, after using the locking compression plate treatment method and the simple plate and screw internal fixation treatment method, the results show that: the experimental group patients have shorter recovery time, shorter hospitalization, lower incidence of complication, and the bone's quality of recovery in the patients was higher, which were significantly different from the control group $(\mathrm{P}<0.05)$. It shows that when the treatment method of internal fixation with locking compression steel plate was applied to patients with limb fractures, the wound healing can be promoted, the postoperative complications can be reduced, and the clinical application effects are prominent. As far as the locking compression plate is concerned, it is mainly a new type of fixing material which can fully 
demonstrate the principle of biological fixation in the modern medical field when it is applied, and its advantages can be summarized after application, which is mainly manifested in four aspects ${ }^{[7]}$. First of all, it has the characteristics of minor traumas and etc. Its clinical application in the indirect manual reduction at fracture ends can reduce the damage to the soft tissues. Furthermore, it is unnecessary to cut the periosteum during the surgery, which can preserve the integrity of the bones, promote the recovery of the patients' limb functions to the greatest extent, and is more conducive to promoting the postoperative recovery of the patients ${ }^{[8]}$. Secondly, the co-application of the screw and the steel plate in the affected limb can shorten the time limit and achieve their synergistic effects under the interaction. The patient's stability after the surgery is relatively good and at the same time, it can effectively avoid the problem of fracture displacement in patients during the recovery stage and promote the recovery of patients ${ }^{[9]}$. The fixation effect of the re-locking system is more prominent, it does not need to be inserted close to the bone, and it can reduce the compression of the periosteum to ensure the blood supply to the fracture site and promote its recovery. Finally, when the compression steel plate is applied, its unique design can fully reflect the biomechanics. When compared to ordinary steel plates, its corresponding firmness and stability are stronger, which can effectively reduce infection problems, skin necrosis and other conditions which reduce the incidence of complications in patients ${ }^{[10]}$. In short, the application of the locking compression plate method can effectively protect the periosteum and soft tissues at the fracture ends, reduce the blood flow at the fracture and accelerate the healing of the fracture, showing prominent treatment efficacy.

In conclusion, when the locking compression plate treatment method is applied to patients with limb fractures, the prognostic effects are more prominent, the clinical application is safe, it is of positive significance for the recovery of patients and can be promoted.

\section{References}

[1] Zhao P. Evaluation on the efficacy of interlocking intramedullary nail in the treatment of bone nonunion after traumatic fracture of limbs [J]. Journal of North Sichuan Medical College, 2018.

[2] Li J, Jiang C, Zhu YS. Comparison of the effects of closed reduction with interlocking intramedullary nail and percutaneous minimally invasive locking compression plate in the treatment of tibial fractures $[\mathrm{J}]$. Chinese Remedies \& Clinics, 2018(6).

[3] Sun MK. Clinical application of locking compression plate fixation in treatment of limb fractures [J]. Journal of Hunan University of Chinese Medicine, 2018(A01):58-59.

[4] Liu XJ. The effect of minimally invasive percutaneous locking compression plate internal fixation on postoperative visual analogue score and fracture healing time in patients with limb fractures $[\mathrm{J}]$. Shanxi Medical Journal, 2019, 048(005):593-595.

[5] Zhang H, Gai W. Clinical application of minimally invasive percutaneous plate osteotomy combined with locking compression plate internal fixation in patients with extremities fracture [J]. Journal of Guangxi Medical University, 2019, 036(004):613-617.

[6] Chang T, Song JJ, Qi TN. Effect of minimally invasive percutaneous locking compression plate internal fixation for extremity fractures on patients' bone mineral density and bone metabolism [J]. Chinese Journal For Clinicians, 2020(11).

[7] Zhang T, Zhang HJ. Comparison of the effects of bridging combined internal fixation and locking compression plate internal fixation in the treatment of limb fractures $[\mathrm{J}]$. Journal of Hunan Normal University (Medical Sciences), 2018.

[8] Zhang T, Zhang HJ. Comparison of the effects of bridging combined internal fixation and locking compression plate internal fixation in the treatment of limb fractures [J]. Journal of Hunan Normal University (Medical Sciences), 2018, v.15;No.62(03):176-179.

[9] Feng J. Effect comparison of interlocking intramedullary nails and locking compression plates in treating femoral shaft fracture [J]. Journal of Clinical Orthopaedics, 2018, 21(06):82-84.

[10] Bai ZJ. Analysis of the clinical application of locking compression plate internal fixation in the treatment of limb fractures [J]. Chinese Remedies \& Clinics, 2019. 